\title{
MORBIDADE HOSPITALAR POR ACIDENTE DE TRÂNSITO EM PERNAMBUCO
}

\author{
Hospitalar Morbidity In Traffic Accident In Pernambuco \\ Morbilidad Hospitalaria En Accidente De Tráfico En Pernambuco
}

Flávia Emília Cavalcante Valença Fernandes ${ }^{1}$, Layanna Dryelle da Silva Vasconcelos ${ }^{2}$, Maria Elda Alves de Lacerda Campos³.

Resumo: O estudo objetivou avaliar a morbidade hospitalar por acidente de trânsito, em Pernambuco, segundo sexo e faixa etária, entre 2007 e 2012. Realizou-se estudo quantitativo descritivo referente às internações por acidente de trânsito. Os dados foram tabulados por meio do Sistema de Informações Hospitalares do SUS, tratados no programa estatístico gretl e Stata12. A pesquisa foi aprovada no Comitê de Ética, sob o no CAAE: 36271414.9.0000.5207. A taxa de internação apresentou uma média de 43,33 /100 mil para os homens e de 9,67 /100 mil para as mulheres, sendo a faixa etária entre 20 e 29 anos a mais acometida para ambos os sexos. Nos idosos, a taxa de internação passou de 8,03/100 mil, em 2007, para 45,09/100 mil, em 2012. A ocorrência diferenciada das taxas de internações, por faixa etária, evidenciou significativa participação dos homens, jovens e das mulheres idosas nos acidentes de trânsito, em Pernambuco. Em conclusão, a morbidade hospitalar por acidente de trânsito cresceu, havendo variação entre grupos etários e sexos, com destaque para jovens e idosos. Os resultados confirmaram a necessidade de controlar a

\footnotetext{
${ }^{1}$ Doutoranda em Inovação Terapêutica (UFPE). Mestre em Gestão e Economia da Saúde pela Universidade Federal de Pernambuco (UFPE). Atualmente é Professora Assistente da Universidade de Pernambuco Campus Petrolina na área de Saúde Coletiva, Colegiado de Enfermagem. Universidade de Pernambuco, e-mail: flavia.fernandes@upe.br. http://orcid.org/0000-0003-2840-8561 http://lattes.cnpq.br/0695657330429678.

${ }^{2}$ Enfermeira pela Universidade de Pernambuco, e-mail: layvasconcelos@ hotmail.com. http://orcid.org/0000-0003-2548-9175, http://lattes.cnpq.br/3502838814672113.

${ }^{3}$ Mestre em Vigilância sobre Saúde (UPE). Professora Assistente do Colegiado de Enfermagem UPE Campus Petrolina. E-maill: elda.campos@upe.br. http://orcid.org/0000-0001-8648-4795, http://lattes.cnpq.br/9910533271487153.

Rev. Gestão \& Saúde (Brasília) Vol. 10, n. 01, Jan. 2019.
}

ocorrência desses eventos e sugerem maior aprofundamento no estudo.

Palavras chave: Morbidade; Acidentes de trânsito; Hospitalização.

Abstract: The objective of this study was to evaluate hospital morbidity due to traffic accident in Pernambuco between 2007 and 2012, grouping the results by sex and age. A descriptive quantitative study was carried out, being data retrieved from SUS' Hospital Information System and treated by means of the statistical program gretl and Stata12. The research was approved by the Ethics Committee under the number CAAE: 36271414.9.0000.5207. The average hospitalization rate for men was 43.33 per 100 thousand and of 9.67 per 100 thousand for women, being the age group between 20 and 29 years old the most affected for both sexes. As for the elderly, the hospitalization rate rose from 8.03 per 100 thousand in 2007 to 45.09 per 100 thousand in 2012. The variation in hospitalization rates by age group evidenced a significant participation of men, young people and elderly women, allowing to conclude that hospital morbidity due to traffic accident not only increased but also affected young and elderly people. The results not only confirm the need to control the occurrence of these events but also suggest further studies.

Keywords: Morbidity; Traffic accidents; Hospitalization.

\section{Introdução}

Os acidentes fazem parte do grupo das causas externas de acordo com a $10^{\circ}$ Classificação Internacional de Doenças - CID10, da Organização Mundial de Saúde (OMS), e 
provocam um alto impacto na sociedade e nas economias nacionais $(1,2)$. Compreendem episódios não propositais e evitáveis, capazes de gerar danos e/ou ferimentos, que podem levar à morte a pessoa que sofreu o trauma(3). Dentre o grupo dos acidentes, destacam-se os de transporte, entendidos como aqueles que com veículo em via pública, excluindo os acidentes por água e os de transporte aéreo ou espacial(3).

A cada ano, contabiliza-se mais de um milhão de mortes por AT, atingindo, principalmente, a faixa etária de 15 a 29 anos (1). Ademais, aproximadamente 20 milhões de pessoas sofrem lesões decorrentes desses acidentes, algumas das quais se tornam permanentemente incapacitadas, necessitando de hospitalização ou tratamento médico especializado(4,5).

Essa conjuntura aponta para uma séria epidemia no trânsito das vias públicas, principalmente nos países em desenvolvimento. $\mathrm{O}$ fato pode ser atribuído ao desequilíbrio entre a elevação célere da taxa de motorização e a deficiência de investimentos nas ações intersetoriais, comum em muitos países em desenvolvimento(6).

Estudos nessa área, voltados para o estado de Pernambuco, que avaliam esses eventos para todas as faixas etárias e sexos, nas internações hospitalares, ainda são escassos. Esta realidade despertou interesse em desenvolver um estudo para avaliar a morbidade hospitalar por acidente de trânsito, no estado de Pernambuco, no período de 2007 a 2012, por sexo e faixa etária.

\section{Métodos}

A pesquisa foi realizada no estado de Pernambuco, localizado no centro-leste da região Nordeste do Brasil, apresentando uma população de 8.796 .448 , em 2010(7). Trata-se de um estudo quantitativo descritivo, cuja amostra foi composta pelas internações hospitalares por acidente de trânsito de residentes no estado, e que ocorreram no período de 2007 a 2012.

Foram utilizadas as definições estabelecidas pela Classificação Internacional de Doenças, envolvem um veículo, sobretudo os empregados para o deslocamento de pessoas ou mercadorias, usado no momento da ocorrência. O acidente de trânsito (AT) está inserido no grupo de acidentes de transporte, e é aquele que ocorre

em sua $10^{\mathrm{a}}$ Revisão (CID-10), para os AT incluídos nas categorias V01 a V89(8). As fontes dos dados foram: o Sistema de Informações Hospitalares (SIH) e censos e estimativas populacionais do Instituto Brasileiro de Geografia e Estatística (IBGE), disponíveis no sítio eletrônico do DATASUS, por meio do Tabnet.

Foram estudadas as seguintes variáveis: sexo, faixa etária detalhada a cada 10 anos e grupo etário com agregação em crianças (menores de 20 anos), adultos (entre 20 e 50 anos) e idosos (60 anos ou mais). A morbidade hospitalar por AT foi coletada a partir de informações das Autorizações de Internações Hospitalares (AIH) aprovadas no SIH, sendo consideradas apenas as informações ocorridas no Sistema Único de Saúde (SUS).

A coleta dos dados do SIH ocorreu em dezembro de 2014 e janeiro de 2015, tendo sido realizada em duas etapas, uma para o ano de 2007 e outra para o período entre 2008 e 2012. Justifica-se essa divisão devido ao ano de implementação da Tabela Unificada do SUS (Tabela de Procedimentos, Medicamentos, Órteses, Próteses e Materiais Especiais do SUS). Essa divisão não interferiu na análise dos dados, visto que foi utilizada a CID-10 e não os códigos dos procedimentos.

Os dados foram tratados a partir das medidas de tendência central (média) e dispersão (desvio padrão), intervalo de confiança e foram calculadas as taxas por AT, sendo analisada a evolução destas, no período(9).

Os intervalos de confiança das taxas foram calculados para comparação entre os sexos e faixas etárias no período estudado. O intervalo de confiança, obtido para cada taxa, foi calculado assumindo-se a distribuição de Poisson para taxas ao nível de confiança de $95 \%$. 
Os dados coletados foram tabulados e apresentados na forma de gráficos e tabelas através do Microsoft Excel 2013 e tratados no programa estatístico gretl versão 2015d e Stata 12.

O estudo integra a pesquisa: "Acidentes de trânsito: avaliação da morbimortalidade e seus custos hospitalares em Pernambuco" e foi aprovado pelo Comitê de Ética em Pesquisa com Seres Humanos da Universidade de Pernambuco, sob o $\mathrm{n}^{\circ}$ CAAE: 36271414.9.0000.5207. O mesmo obedeceu aos aspectos éticos determinados pela resolução nº466/2012 do Conselho Nacional de Saúde (CNS)(10).

\section{Resultados}

Os acidentes nas vias públicas foram responsáveis por um total de 13.758 internações, entre 2007 e 2012. Observou-se uma diferença importante na taxa de internação hospitalar entre o sexo masculino e feminino. Os homens foram responsáveis por uma taxa média de 43,33/100.000 (IC95\% 38,23 - 48,93), enquanto as mulheres apresentaram uma taxa média de 9,67/100.000 (IC95\% 7,34 - 12,50) (Tabela $1)$.

$\mathrm{Na}$ população do sexo masculino, os jovens foram os mais acometidos, apresentando taxa média de internação nas faixas etárias entre 20 e 29 anos (80,50/100.000; IC $95 \% 73.48$ $88.01)$ e entre 30 e 39 anos (71,00 IC95\% 64,42 - 78.07). Chamou a atenção a taxa de internação em menores de 1 ano $(12,33 / 100.000$; IC95\% 9,68 - 15.48), que superaram as internações nas faixas etárias entre 1 e 9 anos (5,33/100.000; IC 05\% 3,65 7.53) e na população idosa com mais de 80 anos $(11,50 / 100.000 ;$ IC95\% 8,95 - 14.55) (Tabela 1).

A população feminina, na faixa etária entre 20 e 29 anos, também foi a mais acometida $(13,17 / 100.000 ; \quad$ IC95\% 10,42 - 16.41), seguida da população idosa com mais de 80 anos (12,5/100.000; IC95\% 9,83 - 15.67) e entre 70 e 79 anos (11,83/100.000; IC95\% 9,24 - 14.93).

Analisando-se as taxas médias de internações entre os ciclos da vida de homens e mulheres, observamos comportamento semelhante com o predomínio dos adultos, seguidos dos idosos e crianças. As taxas do sexo masculino predominaram em relação às mulheres, com taxas médias de 64,67/100.000 (IC95\% 58,39 - 71.43) e 11,50 (IC95\% 8,95 - 14.55), respectivamente, nos adultos. Em homens idosos ocorreram, em média, 21,83 internações por 100.000 habitantes (IC95\% 18,25 - 25.91), em contraposição às 11,33/100.000 internações femininas (IC95\% 8,5 - 14.37) (Tabela 1). As crianças do sexo masculino (16,83/100.000; IC95\% 13,71 20.45) também apresentaram uma taxa de internação média superior às crianças do sexo feminino $(6,00 / 100.000$; IC95\% 4,20 - 8.31) (Tabela 1).

Tabela 1 - Taxa de Internação por acidente de trânsito geral, por faixa etária e sexo, Pernambuco, 2007 a 2012 (N=13.758).

\begin{tabular}{llllllllllll}
\hline & \multicolumn{3}{c}{ Sexo masculino } & \multicolumn{5}{c}{ Sexo feminino } \\
\hline Taxa de Internação* & Obs & Média & $\begin{array}{l}\text { Erro } \\
\text { padrão }\end{array}$ & IC (95\%)** & Obs & Média & $\begin{array}{l}\text { Erro } \\
\text { padrão }\end{array}$ & IC (95\%)** \\
\hline Taxa de Internação geral & 6 & 43.33 & 2.69 & 38.23 & 48.93 & 6 & 9.67 & 1.27 & 7.34 & 12.50 \\
\hline Faixa etária & & & & & & & & & & \\
\hline Menores de 1 ano & 6 & 12.33 & 1.43 & 9.68 & 15.48 & 6 & 5.67 & 0.97 & 3.92 & 7.92 \\
Taxa de internação 1 a 9 anos & 6 & 5.33 & 0.94 & 3.65 & 7.53 & 6 & 4.00 & 0.82 & 2.56 & 5.95 \\
Taxa de internação 10 a 19 anos & 6 & 26.50 & 2.10 & 22.54 & 30.95 & 6 & 7.50 & 1.12 & 5.47 & 10.04 \\
Taxa de internação 20 a 29 anos & 6 & 80.50 & 3.66 & 73.48 & 88.01 & 6 & 13.17 & 1.48 & 10.42 & 16.41 \\
Taxa de internação 30 a 39 anos & 6 & 71.00 & 3.44 & 64.42 & 78.07 & 6 & 11.67 & 1.39 & 9.09 & 14.74
\end{tabular}


Fernandes FECV, Vasconcelos LDS, Campos MEAL.

Taxa de internação 40 a 49 anos 6

Taxa de internação 50 a 59 anos 6

Taxa de internação 60 a 69 anos 6

Taxa de internação 70 a 79 anos 6

Taxa de internação 80 anos ou

mais

Grupos etários

Taxa de internação em crianças 6

Taxa de internação em adultos 6

Taxa de internação em idosos 6
$50.67 \quad 2.91$

$36.67 \quad 2.47$

$25.67 \quad 2.07$

$19.67 \quad 1.81$

$6 \quad 11.50 \quad 1.38$

\begin{tabular}{lllllll}
45.13 & 56.69 & 6 & 9.33 & 1.25 & 7.05 & 12.12 \\
31.98 & 41.85 & 6 & 10.83 & 1.34 & 8.36 & 13.81 \\
21.77 & 30.06 & 6 & 10.83 & 1.34 & 8.36 & 13.81 \\
16.28 & 23.55 & 6 & 11.83 & 1.40 & 9.24 & 14.93 \\
8.95 & 14.55 & 6 & 12.50 & 1.44 & 9.83 & 15.67 \\
\hline
\end{tabular}

*Taxa por 100.000 habitantes. assumindo distribuição de Poisson para taxas.

Fonte: Sistema de Informações Hospitalares (SIH) e Instituto Brasileiro de Geografia e Estatística (IBGE).

Analisando a evolução da taxa de internação, ao longo do período, na população feminina, observou-se- uma redução entre 2007 e 2008, em todos os grupos etários. Nas crianças e nos adultos, a evolução se apresentou sempre crescente após 2008, passando de 2,46/100.000, em 2007, para
15,06/100.000, em 2012, nas crianças, e de $3,94 / 100.000$ para 23,73/100.000, em 2012, nos adultos. As idosas apresentaram crescimento da taxa média de internação entre 2008 e 2011, quando apresentaram redução. Em todo o período, a taxa passou de 9,11, em 2007, para 18,34/100.000, em 2012 (Figura 1)

Figura 1 - Evolução da taxa de internação na população feminina segundo grupo etário, Pernambuco, 2007 a 2012.

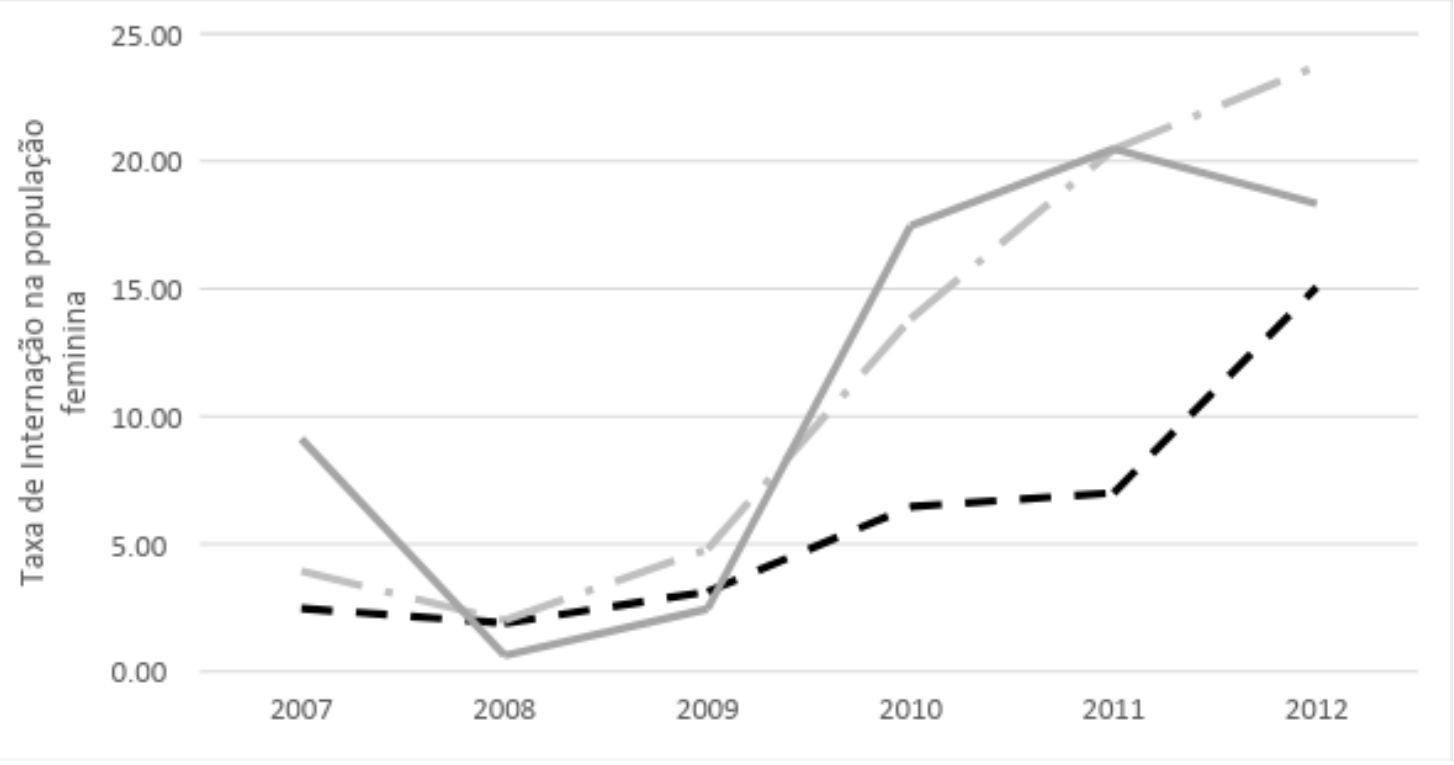

*Taxa por 100.000 habitantes.

Fonte: SIH/SUS e IBGE

A evolução da taxa de internação nos homens também se apresentou crescente após 2008. Assim como na população feminina, houve 
uma redução entre 2007 e 2008. Nas crianças, houve um crescimento de 5,79, em 2007, para 39,04/100.000, em 2012. Os adultos apresentaram internação de 12,09/100.000, passando para 45,09/100.000, em 2012 (Figura 2).

Figura 2 - Evolução da taxa de internação na população masculina segundo grupo etário, Pernambuco, 2007 a 2012. em 2007, passando para 142,87 internações por 100.000 habitantes, em 2012. Os idosos apresentaram taxa de internação de 8,03/100.000, em 2007,

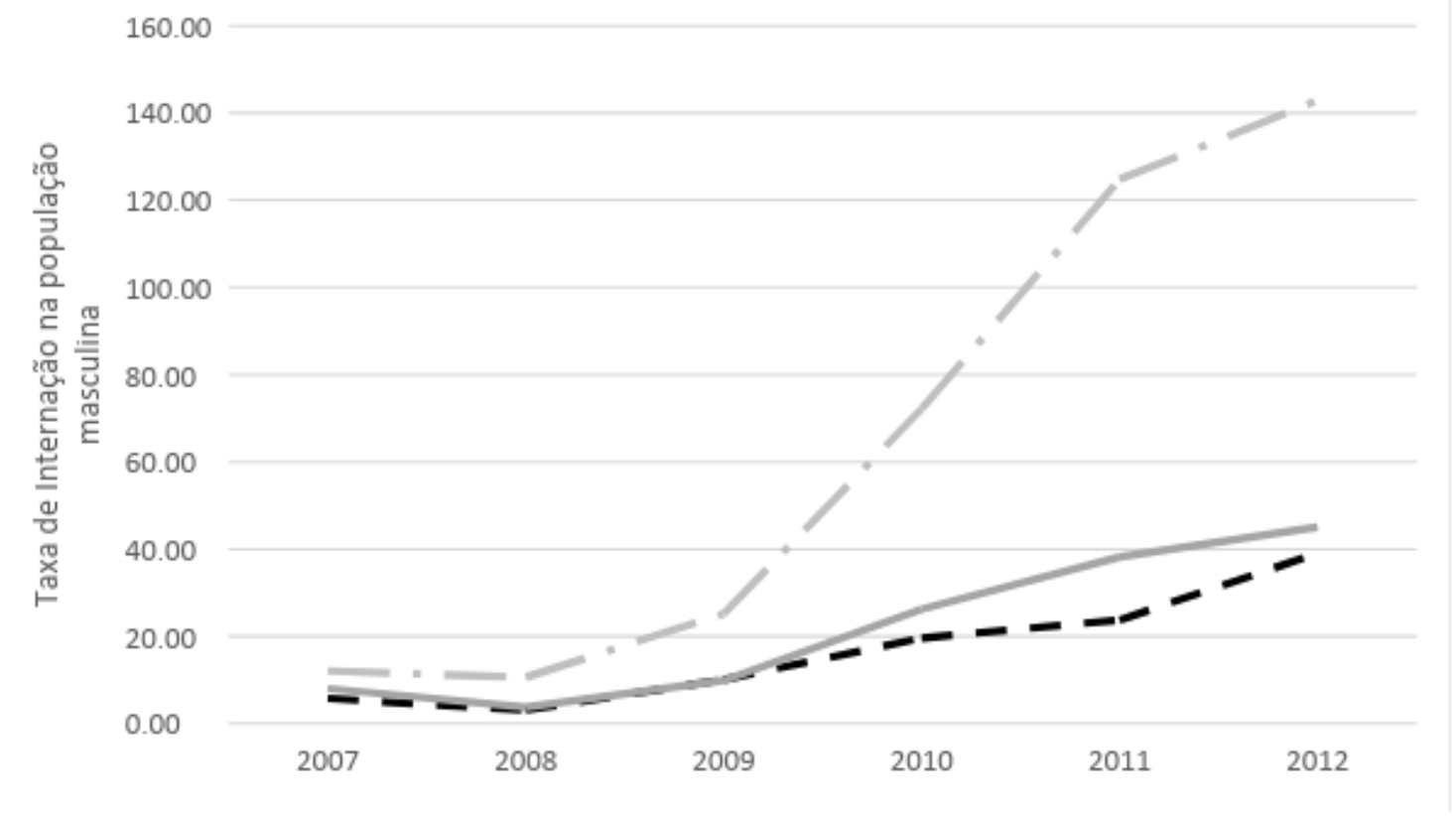

*Taxa por 100.000 habitantes.

Fonte: SIH/SUS e IBGE

\section{Discussão}

O presente estudo apontou uma ocorrência de internação por acidentes de trânsito mais evidente nos homens em relação às mulheres.

Estudo realizado em um hospital de emergência, em Santa Catarina, apontou, também, uma ocorrência de acidentes de trânsito superior nos homens em relação às mulheres, com 78,2\% dos acidentes registrados $^{(11)}$. Estudos também corroboram os resultados encontrados na pesquisa, apontando a ocorrência do sexo masculino sempre superior a ocorrência do sexo feminino $^{(5,12)}$.

Esses resultados podem estar relacionados à grande exposição do homem jovem no trânsito e por comportamentos que o faz assumir maiores riscos na condução de veículos $^{(13)}$. Um estudo sobre o perfil das vítimas de acidentes motociclísticos, em Teresina - PI, referiu à imaturidade, busca de emoções, a tendência de superestimar suas capacidades, impulsividade e uso de álcool e/ou drogas como fatores que contribuem para a maior incidência de AT nesse grupo ${ }^{(14)}$.

A proporção de pessoas jovens internadas prevaleceu no presente estudo, em especial na faixa etária de 20 a 29 anos. Estudos acerca dos acidentes de trânsito também embasam o resultado alcançado, tanto relacionado a morbidade hospitalar quanto a mortalidade ${ }^{(6,12,15)}$. Outras regiões do mundo, como a região africana, também sofrem com os elevados índices de acidentes 
de trânsito, tendo como pessoas mais acometidas, a população jovem e do sexo A ocorrência diferenciada por faixa etária na população feminina evidenciou a significativa participação da população idosa nos acidentes de trânsito, em Pernambuco, em especial, as mulheres mais idosas, ou seja, com mais de 80 anos de idade.

Tal achado também foi semelhante à análise realizada pela Organização Mundial da Saúde para a região africana, a qual apresentou uma taxa de mortalidade para pessoas com mais de 60 anos superior às taxas na faixa etária mais jovem, quando analisada na população feminina ${ }^{(5)}$.

A participação dos idosos nos acidentes de trânsito está relacionada, na literatura, aos acidentes com pedestre ${ }^{(16)}$. O aumento do número dessa população e a melhora da qualidade de vida, inclusive com a realização de atividade profissional, pode estar proporcionando maior exposição e, consequentemente, possibilitando o aumento da ocorrência de trauma por AT neste grupo $^{(17)}$.

Uma pesquisa sobre os acidentes de trânsito, ocorridos de 1998 a 2010, constatou que, no país, o perfil dos eventos por causas externas, no idoso, difere em alguns aspectos encontrados entre os mais jovens, apresentando uma maior predisposição aos atropelamentos, chamando a atenção para a elevada proporção de mortes por esta causa $(48,2 \%)^{(16)}$.

Observou-se, também, crescimento das taxas de internação após o ano de 2008. A diminuição das taxas entre 2007 e 2008 pode estar relacionada a mudança na codificação dos procedimentos hospitalares do SUS, o que pode ter gerado distorção na produção hospitalar. Assim, a atenção deve ser voltada principalmente após 2008. A população masculina apresentou evolução da taxa em todos os grupos etários como crianças, adultos e idosos. Já nas mulheres, o comportamento da taxa no grupo de idosas foi de involução entre 2011 e 2012.

Tal fato é corroborado pelo Mapa da Violência 2013, revelando que os AT crescem $\operatorname{masculino}^{(5)}$.

das taxas de internações desde 1998, com aumento mais expressivo nas taxas de internação a partir de 2009, principalmente entre os motociclistas ${ }^{(3)}$.

As estimativas da OMS apontam para a tendência de crescimento ao longo dos anos. Fato atribuído ao desequilíbrio entre a elevação da taxa de motorização e a deficiência de investimentos nas vias públicas $^{(6)}$.

Além dos dados encontrados nos idosos, atenção importante deve ser dada também a morbidade por AT em menores de 1 ano, como revelou o estudo sobre a vigilância de violências e acidentes no país, em que as crianças (0 a 9 anos) e idosos apresentaram, proporcionalmente, maior frequência entre os atendimentos por acidentes $(11,8 \%$ e $8,8 \%)$ do que por violências $(4,7 \% \text { e } 3,8 \%)^{(18)}$.

A morbidade por AT inclui lesões em variados graus de extensão e magnitude, incapacidades permanentes e temporárias, sequelas, dor e sofrimento para as vítimas e seus familiares em todo o mundo. Esses acidentes provocam danos de gravidade, acometendo preferencialmente homens jovens em idade produtiva ${ }^{(15)}$.

\section{Conclusão}

A morbidade hospitalar, no estado de Pernambuco, apresentou os homens como as vítimas mais atingidas em relação às mulheres. A faixa etária de 20 a 29 anos foi a mais acometida em ambos os sexos. Contudo, a ocorrência de elevadas taxas de internação em idosas foi identificada na análise. Nas mulheres, esse grupo etário também apresentou tendência de decréscimo após 2011.

A variação da taxa de internação, ocorrida entre 2007 e 2008, pode estar relacionada a mudanças nos códigos de cobranças dos procedimentos em decorrência da mudança na Tabela Unificada do SUS para lançamento das informações no Sistema de 
Informações Hospitalares do SUS, o que pode ter gerado distorções.

Apesar dos resultados apresentados, a pesquisa apresenta algumas limitações que podem intervir na quantidade e qualidade dos resultados, uma vez que os dados foram extraídos a partir de fontes secundárias, que dependem do preenchimento primário das autorizações de internações hospitalares. Contudo, isso não indica a inviabilidade dos resultados apresentados.

Espera-se que o presente estudo possa subsidiar gestores na formação de políticas que sejam capazes de minimizar tanto o número de mortes quanto de internações desinentes dos acidentes de trânsito.

\section{Referências}

1. Who. Injuries and Violence: The Facts. Geneva, Switz WHO [Internet]. 2010;2-18. Available from: http://scholar.google.com/scholar?hl=en\&btn $\mathrm{G}=$ Search\&q=intitle:Injuries+and+Violence:+ The+Facts\#0

2. Waiselfisz JJ. Os jovens do Brasil: Mapa da violência 2014. Rio de Janeiro: Qualidade; 2014.

3. Waiselfisz JJ. Mapa da violência 2013: acidentes de trânsito e motocicletas [Internet]. Rio de Janeiro: CEBELA: Centro Brasileiro de Estudos Latino-Americanos; 2013. p. 96. Available from: http://www.mapadaviolencia.org.br/pdf2013/ mapa2013_transito.pdf

4. Mohan D. Road traffic injuries: a neglected pandemic [Internet]. 2003. Available from: http://who.int/bulletin/volumes/81/9/Mohan.p df

5. Mohan D, Tiwari G, Khayesi M, Nafukho FM. Road traffic injury prevention: training manual. World Heal Organ [Internet]. 2006; Available from: http://apps.who.int/iris/bitstream/10665/43271 /1/9241546751_eng.pdf

6. World Health Organization. Supporting a decade of action. World Health Organization. 2013.
7. IBGE IB de G e E. Censo populacional 2010 [Internet]. 2010. Available from: http://www.ibge.gov.br/estadosat/perfil.php?si gla $=$ pe

8. Brasil MDS. Agrupamentos da Classificação Internacional da Doença 10a Revisão (CID 10) [Internet]. 2015. Available from: http://www2.datasus.gov.br/cid10/V2008/We bHelp/v01_y98.htm

9. Barros SG De, Chaves CL. A utilização do Sistema de Informações Ambulatoriais ( SIASUS ) como instrumento para caracterização das ações de saúde bucal. Epidemiol e Serviços Saúde [Internet]. 2003;12(1):41-51. Available from: http://scielo.iec.pa.gov.br/scielo.php?pid=S16 79-49742003000100005\&script=sci_arttext

10. CNS CN de hoje. Resolução 466 do Comité de Ética. 2012.

11. Trevisol DJ, Bohm RL, Vinholes DB. Perfil epidemiológico dos pacientes vítimas de acidentes de trânsito atendidos no serviço de emergência do Hospital Nossa Senhora da Conceição em Tubarão, Santa Catarina. Sci Med (Porto Alegre) [Internet]. 2012;22(3):148-52. Available from: http://revistaseletronicas.pucrs.br/ojs/index.ph p/scientiamedica/article/viewFile/10823/8185

12. Oliveira APP, Abreu ÂMM, Paixão LAR. Possível impacto da "lei seca" nos atendimentos a vítimas de acidentes de trânsito em uma unidade de emergência. Esc Anna Nery [Internet]. 2013;17(1):54-9. Available from: http://www.scielo.br/pdf/ean/v17n1/08.pdf

13. Bastos YGL, Andrade SM, Soares DA. Características dos acidentes de trânsito e das vítimas atendidas em serviço pré-hospitalar em cidade do Sul do Brasil , 1997 / 2000. Cad Saude Publica [Internet]. 2005;21(3):815-22. Available from: http://www.scielo.br/pdf/csp/v21n3/15.pdf

14. Gomes S da L, Santos YA, Dourado SBPB, Coêlho DMM, Moura MEB. Perfil das vítimas de acidentes motociclísticos admitidas nas terapias intensivas de um hospital público. Rev enferm UFPE line. 2014;8(7):2004-12.

15. Oliveira LR De, Mello-Jorge MHP de. Análise epidemiológica das causas externas em unidades de urgência e emergência em Cuiabá / Mato Grosso. Rev Bras Epidemiol [Internet]. 2008;11(3):420-30. Available 
from:

http://www.scielo.br/pdf/rbepid/v11n3/08.pdf

16. Bacchieri G, Barros AJD. Acidentes de trânsito no Brasil de 1998 a 2010: muitas mudanças e poucos resultados. Rev Saúde Pública [Internet]. 2011;45(5):949-63. Available from: http://www.scielo.br/pdf/rsp/v45n5/2981.pdf

17. Luz TCB, Malta DC, Sá NNB, Silva MMA, Lima-Costa MF. Violências e acidentes entre adultos mais velhos em comparação aos mais jovens : evidências do Sistema de Vigilância de Violências e Acidentes ( VIVA ), Brasil. Cad Saude Publica [Internet]. 2011;27(11):2135-42. Available from: http://www.scielo.br/pdf/csp/v27n11/07.pdf

18. Brasil MDS. Sistema de Vigilância de Violências e Acidentes (VIVA) [Internet]. Brasília: Ministério da Saúde; 2013. 164 p. Available from: http://bvsms.saude.gov.br/bvs/publicacoes/sist ema_vigilancia_violencia_acidentes.pdf

\section{Participação dos autores}

FERNANDES, FECV atuouna concepção teórica, análise estatística e revisão da redação final do texto;

VASCONCELOS, LDS atuou na concepção teórica, elaboração, coleta de dados, análise estatística e redação final do texto;

CAMPOS, MEAL atou na análise dos dados, na concepção teórica, revisão da redação final.

Recebido: 30.01.2017

Revisado: 31.01.2017

Aprovado: 31.01.2017 